Revista Ingeniería y Región. 2016;15(1): 37.47

\title{
Propuesta metodológica de modelación dinámica de la Huella de Carbono en las labores de campo de un cultivo de caña de azúcar
}

\section{Methodology Proposal for Carbon Footprint Dynamic Modeling at Sugarcane Crops Field Labors}

\author{
Andrés López Astudillo ${ }^{1}$, Lina Marcela Rodríguez ${ }^{2}$, Claudia Lubo $^{3}$, Fernando Arenas $^{4}$ \\ y Beatriz Sierra ${ }^{5}$
}

\begin{abstract}
Resumen
La agricultura contribuye al cambio climático al emitir a la atmósfera aproximadamente el $13.5 \%$ de las emisiones de gases de efecto invernadero (GEI) generadas por el hombre. Esto se presenta como consecuencia tanto de las labores agrícolas mecánicas -responsables de la emisión de dióxido de carbono-, como de la descomposición de los fertilizantes, herbicidas y madurantes aplicados al cultivo -los cuales emiten óxido nitroso principalmente-. El sector agrícola también sufre directamente el efecto del cambio climático, lo cual se refleja en fenómenos meteorológicos como tormentas, inundaciones y sequías, los cuales acortan el periodo de crecimiento de la planta y merman los rendimientos del cultivo. Este artículo describe la metodología identificada en el proyecto para modelar la huella de carbono generada durante la preparación y el levante del cultivo de la caña de azúcar, desde el punto de vista de la dinámica de sistemas. la cual representa una base para la formulación de modelos de simulación de mayor complejidad que permitan evaluar el comportamiento de las emisiones de GEI en el corto y largo plazo y contribuir así, como guía en la ejecución de proyectos externos. La propuesta metodológica tiene el potencial de contribuir al direccionamiento de futuras fases de la investigación actual aplicada en el sector azucarero.
\end{abstract}

Palabras clave: Cambio climático; Huella de Carbono; Gases de efecto invernadero; Cultivo de caña de azúcar; Dinámica de sistemas; Modelación.

\begin{abstract}
1 Universidad Icesi, Santiago de Cali, alopez@icesi.edu.co, $\mathrm{PhD}$ (c)

2 Universidad Icesi, Santiago de Cali, lmrodriguez@icesi.edu.co

3 Universidad Icesi, Santiago de Cali, cmlubo@icesi.edu.co, MsC(c)

4 Universidad Icesi, Santiago de Cali, faarenas@icesi.edu.co, PhD(c)

5 Universidad Icesi, Santiago de Cali, beatriz_sierraruiz@yahoo.es
\end{abstract}

Agriculture contributes to Climate Change by emitting into the atmosphere approximately $13.5 \%$ of emissions of greenhouse gases (GHGs) generated by anthropogenic activities. This situation comes as a result of both mechanical farming - responsible of the carbon dioxide emissions and the decomposition of fertilizers, herbicides and ripening accelerators applied to the crop - primary responsible for nitrous oxide

Recibido: 15 de febrero 2016. Aceptado: 30 de marzo 2016. 
emissions - The agricultural sector is also affected directly by climate change effects, which is reflected in weather events such as storms, floods and droughts, shortening the plant growth period and crop yields. This article describes the methodology identified in the project with the purpose on modeling the carbon footprint generated during land preparation and the sugar cane crop increase from the sight of system dy namics, which represents a basis for the formulation of even more complex simulation models in order to assess the GHG emissions behavior in the short and long term. The given methodology implies a guide for external projects execution and carries a significant potential to address future phases since the current applied research on the sugar cane sector.

Key words: Climate Change; Carbon Footprint; Greenhouse Gases; Sugarcane Crop; System Dynamics; Modeling.

\section{Introducción}

La revolución verde surgió en los años 60 , generando un cambio radical en la producción agrícola a nivel mundial. Ésta consistió en la obtención de variedades agrícolas muy productivas con el uso de tecnologías altamente dependientes y costosas para mejorar el rendimiento del cultivo por superficie (Conway \& Barbie, 1988). A pesar de que se solucionaron problemas de hambrunas en el mundo, la aplicación de fertilizantes químicos, pesticidas, herbicidas y técnicas de riego generaron numerosos impactos ambientales que afectaron de manera irreversible el ecosistema (Mclaughlin, Tiller, et al., 1996). Específicamente, la uniformización en la utilización de semillas y los monocultivos generaron un impacto negativo en la biodiversidad, el uso de plaguicidas y fertilizantes se incrementó de forma abrupta acelerando la salinización de los suelos y generando problemas de eutrofización de los recursos hídricos; así como la compactación del suelo por uso de maquinaria pesaday contaminación del agua por el uso de plaguicidas (Rapal Uruguay, 2010). Algunos de los impactos ambientales más agresivos en las actividades agrícolas dependen en gran medida de la utilización de fertilizantes químicos, el no dejar descansar el suelo, el tipo de labranza utilizado, uso de maquinaria pesada, consumo de combustible por las fuentes móviles, entre otros. Específicamente en el cultivo de caña de azúcar, algunos de los principales impactos ambientales se dan a nivel de compactación del suelo debido al uso de maquinaria agrícola, erosión de suelo, emisiones tóxicas y contaminantes por las labores de quemay reducción de la biodiversidad por la expansión del monocultivo (Zoratto, 2006).

En la Declaración de Rio sobre el Medio Ambiente y Desarrollo, la Conferencia de las Naciones Unidas celebrada en Estocolmo en 1972, definió al medio ambiente de la siguiente forma: "El medio ambiente es el conjunto de componentes físicos, químicos, bio- lógicos y sociales capaces de causar efectos directos o indirectos, en un plazo corto o largo, sobre los seres vivos y las actividades humanas." Dada la complejidad de este sistema, no se puede contemplar desde una perspectiva aislada; se requiere un enfoque holístico para su estudio, teniendo en cuenta las interrelaciones entre variables que lo componen y las repercusiones en cadena que puedan ocasionar cualquier tipo de alteración al medio, lo que se conoce como efecto dominó.

La dinámica de sistemas (DS) desarrollada por Jay W. Forrester en el año 1956, es una disciplina que permite investigar la complejidad de un sistema dinámico (Forrester, 1961). Esta herramienta permite analizary modelar el comportamiento temporal histórico y/o futuro de una situación en un entorno complejo (Liévano \& Londoño, 2012). El enfoque sistémico facilita el análisis de los factores de los bucles de retroalimentación en la relación causa-efecto, en términos de flujos y variables de nivel (depósitos), considerando posibles demoras en el sistema. La DS es ampliamente usada en diferentes contextos (sociales, económicos, ecológicos, organizacionales, etc.), y sirve como soporte para una efectiva toma de decisiones por medio del análisis de escenarios en un entorno de simulación.

Este artículo tiene como objetivo presentar una propuesta metodológica desde el punto de vista de la dinámica de sistemas, y profundizar en el desarrollo de los diagramas causales que describen la relación causa-efecto existente entre la productividad del cultivo de caña de azúcar y el impacto ambiental generado a favor de ésta. De esta forma, se presenta su aplicación en el sector azucarero, con información proveniente de los ingenios azucareros y los centros de investigación ubicados en el departamento del Valle del Cauca, Colombia. Posteriormente, se muestran las conclusiones obtenidas en esta fase de la investiga- 
ción y se hace énfasis en la importancia de abordar problemas como el expuesto en este estudio por medio de un pensamiento holístico de la situación, el cual es posible adquirir gracias a las herramientas de modelación dinámica que nos ofrece la Teoría General de Sistemas, donde parte la base del Pensamiento Sistémico. Contribuyendo además, a cubrir el nivel de insuficiencia en la información existente sobre modelos dinámicos que permitan abordar de forma sistémica la complejidad del impacto ambiental generado por el sector azucarero.

Finalmente, se realiza una introducción de las próximas fases a abarcar, como son el desarrollo de los sub-modelos de emisión en cada una de las etapas de la cadena de producción de caña de azúcar, y su posterior análisis de escenarios.

\section{Metodología}

\subsection{Fases del Proyecto de Investigación}

En primer lugar, se evaluaron las cinco etapas del proceso de modelación dinámica (Sterman, 2000), las cuales son: articulación del problema, hipótesis dinámica, formulación, validación, diseño y evaluación de políticas. A partir de estas etapas, se establecieron las siguientes fases del proyecto como estructura metodológica para abordar el problema de investigación. Vale la pena mencionar que en el presente artículo se profundizará en el desarrollo de los diagramas causales necesarios para diseñar y alimentar los modelos dinámicos de simulación, los cuales se encuentran enmarcados dentro de la fase de diseño de los modelos.

\section{i). Fase de Investigación}

En la primera fase se desarrolló una revisión literaria sobre 55 artículos existentes de modelos de dinámica de sistemas enfocados en evaluar el comportamiento de la huella de carbono en diferentes sectores, principalmente agriculturay energía (López, Rodríguez, Lubo, Arenas, \& Sierra, 2014). Entre los "Vacíos" o deficiencias de información encontradas en la literatura, se observó que no existe suficiente información sobre modelación dinámica de las emisiones generadas en el sector de la agricultura, específicamente en el sector azucarero. Por tanto, se evidenció como oportunidad el abordar la complejidad de esta problemática desde un enfoque de dinámica de sistemas, el cual permitirá una mejor toma de decisiones estratégicas en la agroindustria azucarera desde el punto de vista práctico y metodológico.

Ante la escasez de información en investigaciones previas que permitan visualizar de forma sistémica las consecuencias de este fenómeno global que hoy en día responsabiliza al sector agroindustrial; se definió modelar el comportamiento de los gases de efecto invernadero (GEI) más representativos en el sector azucarero, los cuales son: Dióxido de Carbono $\left(\mathrm{CO}_{2}\right)$ y Óxido Nitroso $\left(\mathrm{N}_{2} \mathrm{O}\right)$, generados por las labores agrícolas mecánicas ejecutadas en las áreas de campo, y las aplicaciones realizadas durante la preparación del suelo y levante de un cultivo de caña de azúcar. Esta modelación se propone como caso de estudio, la cual tiene como propósito determinar la huella de carbono generada por las labores de campo de un cultivo de caña de azúcar, específicamente para la región de Valle del Cauca.

\section{ii). Fase de Diseño de los Modelos}

En primer lugar, esta segunda fase busca articular el problema de investigación, desarrollar las hipótesis dinámicas y formular los diagramas causales, como los requerimientos fundamentales para definir de manera formal los modelos dinámicos de simulación.

En segundo lugar, esta etapa comprende la formulación de los modelos propuestos, con base en la teoría de la dinámica poblacional o demográfica bajo la naturaleza de investigación descriptiva - analítica o explicativa, de acuerdo al proceso de modelación dinámico propuesto por Sterman (2000). Para su desarrollo se utiliza el software Vensim ${ }^{\circledR}$ PLE. Las ecuaciones que alimentan los modelos se obtienen a partir del cálculo teórico de las emisiones de dióxido de carbono $\left(\mathrm{CO}_{2}\right)$ provenientes de la combustión móvil (Waldron et al., 2006) y estacionaria (Gómez $\&$ Watterson, 2006). Así como, el cálculo teórico de las emisiones de óxido nitroso $\left(\mathrm{N}_{2} \mathrm{O}\right)$ de los suelos gestionados y emisiones de dióxido de carbono $\left(\mathrm{CO}_{2}\right)$ derivadas de la aplicación de cal y urea (De Klein et al., 2006). Estos cálculos se deben adaptar a partir de las ecuaciones establecidas por las directrices del Panel Intergubernamental del Cambio Climático (IPCC) de 2006.

\section{iii). Fase de Validación}

Dada la importancia de los modelos de simulación en la toma de decisiones estratégicas a un menor costo y riesgo por tratarse de un entorno virtual, se debe 
validar si éste provee una representación adecuada del sistema real. La validación se debe realizar en la estructura y nivel de comportamiento del sistema. En primer lugar, se debe hacer una evaluación cualitativa de relaciones entre las variables, basado en un conocimiento probado del sistema modelado. De manera consecuente, se deben realizar pruebas del comportamiento a los patrones generados por los modelos. Sterman (2000) propone varias pruebas para validar la estructura y el comportamiento simulado de los modelos.

A partir de lo anterior, la evaluación cualitativa de los modelos se debe realizar por medio de la colaboración conjunta de personal académico experto en modelación dinámica y con conocimiento y experiencia en el cálculo de la huella de carbono en el sector azucarero, además de personal con experticia en las labores agrícolas requeridas en campo para la producción de caña de azúcar. Posteriormente, es necesario realizar la evaluación cuantitativa por medio de análisis de sensibilidad. Para esto, se deben establecer supuestos para cambiar el valor de los indicadores y parámetros pertenecientes a los modelos, y observar si se presentan cambios significativos en el comportamiento de los modelos a simular.

\section{iv). Fase de Análisis de Resultados}

El objetivo de esta fase es desarrollar escenarios de simulación de las emisiones generadas en un cultivo de caña comercial, en un cultivo en periodo de transición a orgánico y en un cultivo orgánico. Las simulaciones del modelo de combustión fósil se han proyectado a 25 años, con el propósito de observar un comportamiento de las emisiones de $\mathrm{CO}_{2}$ en el largo plazo, es decir, durante varios ciclos de cosecha del cultivo, como son plantilla ${ }^{6}$ soca ${ }^{7}$. Donde el ciclo tipo plantilla hace referencia a la renovación de un ciclo tipo soca, lo cual ocurre en promedio cada 5 años. Y las simulaciones del modelo por descomposición de los fertilizantes aplicados al suelo se han proyectado a un periodo más corto correspondiente a 5 años, con el fin de evaluar el comportamiento de las emisiones de $\mathrm{N}_{2} \mathrm{O}$ según la dinámica de algunas variables de precipitación y transformaciones de nitrógeno orgánico en mineral que sólo pueden ser observadas en lapsos de tiempo relativamente cortos, dada una acelerada velo- cidad en las reacciones ocurridas en suelo y que en muchos casos por su nivel de complejidad y detalle micro pueden ser despreciadas.

Como resultado, esta fase permite comparar el comportamiento de las emisiones de $\mathrm{CO}_{2}$ generadas durante la preparación del suelo del cultivo en renovación que requiere un número mayor de labores mecánicas y tiempo de preparación del mismo, en contraste con el cultivo tipo soca que ya tiene varias cortes, es decir, que ya ha recibido al menos una preparación de suelo en la corte anterior. Por tanto, este último ciclo requiere un menor número de labores, lo que significa un menor consumo de combustible, y por ende una menor generación de emisiones a la atmósfera. Por otro lado, es posible visualizar el comportamiento de las emisiones de $\mathrm{CO}_{2}$ generadas por las labores mecánicas de levantey mantenimiento del cultivo, además de los riegos requeridos; así como, realizar un contraste de las emisiones de $\mathrm{N}_{2} \mathrm{O}$ generadas por la aplicación de agro-químicos en un cultivo comercial, en transición y orgánico, teniendo en cuenta el ciclo del nitrógeno (emisiones y absorciones en el sistema atmósfera-suelo-planta).

Por otro lado, de acuerdo con Martínez y Londoño (2013), en torno al modelamiento de sistemas, existen tres principios básicos del pensamiento sistémico: la articulación del problema, el análisis de los sistemas y el uso de modelos por medio de la aplicación de diversas herramientas en ingeniería (diagramas causales, arquetipos, análisis de complejidad, etc.). En primer lugar, se deben establecer las hipótesis dinámicas necesarias para identificar las relaciones causales existentes entre las variables definidas a partir de los hallazgos teóricos y experimentales obtenidos en la investigación. Estas hipótesis dinámicas permiten la construcción de los diagramas causales, por medio de los cuales se explica el comportamiento observado de la situación problema, junto con las relaciones causales de retroalimentación positiva (ciclo reforzador) o negativa (ciclo compensador) entre las variables que conforman la estructura del sistema dinámico.

Dada la importancia de los diagramas causales para una formulación adecuada de los modelos dinámicos de simulación, en la tercera sesión se presenta una aplicación de la situación problema global y local des-

6 Ciclo de cosecha que hace referencia a la primera plantación de cultivo, la cual se realiza sobre un terreno que ha sido completamente renovado.

7 Ciclo de cosecha que hace referencia a la plantación realizada a partir del primer corte de caña de azúcar. 
crito en la introducción del presente artículo. Es necesario mencionar que la caracterización inicial del problema de investigación, elaboración de las hipótesis dinámicas y construcción de los diagramas causales, involucro la participación de expertos en el campo académico, visitas de campo a un ingenio azucarero de la región, y otras fuentes secundarias (publicaciones, datos históricos, trabajos previos realizados sobre este caso de estudio, etc.).

\subsection{Delimitación del alcance de estudio}

El Valle Geográfico del río Cauca cuenta con 33 grupos homogéneos de suelos (definidos por la familia textural, el régimen de humedad y la profundidad efectiva) y seis grupos de humedad (definidos por el balance hídrico, la permeabilidad del suelo, la pendiente, los signos de mal drenaje y su profundidad) (Carbonell $e t$ al., 2011). A partir de esta información, se definieron las zonas agroecológicas (ZAE) sobre las cuales se debe hacer un manejo agronómico del cultivo. Para el presente caso de estudio realizado sobre el cultivo de caña de azúcar, es necesario tener en cuenta las zonas de mayor representatividad en el departamento, como son las ZAE 6H1 y 11H1 (Carbonell et al., 2011). Por otro lado, con respecto al cultivo en estudio es necesario mencionar que el proceso de producción de la caña de azúcar depende tanto de las labores de campo, como cosecha del cultivo. Las labores de campo incluyen básicamente la preparación de los suelos y los procesos de levante del cultivo que se requieren según la edad de la planta. Las labores de cosecha por su parte, abarcan la aplicación de madurantes y los procesos de corte, alce y transporte hacia la fábrica de azúcar. El alcance de este estudio cubre las labores mecánicas de preparación de suelos, las cuales dependen del ciclo de cosecha del cultivo. Dado que si éste se encuentra en el ciclo tipo Plantilla, se deben desarrollar seis tipos de labores agrícolas: descepada, subsolada, cincelada, rastro-arado, rastrillada y surca$\mathrm{da}$, las cuales, dependiendo del orden y la intensidad con que se apliquen, conforman seis tipos diferentes de preparaciones de suelo, referenciadas por (Concha $\&$ Rojas, 2011). A su vez, estos se clasifican en tipos de preparación convencionales, los cuales requieren mayor maquinaria y ocho (8) pases en promedio; y tipos de preparación livianos, con un menor número de labores y un promedio de seis (6) pases como máximo. Por su parte, el ciclo de cosecha tipo Soca involucra actividades como encalle de residuos, subsolado y cultivo-aporque, las cuales tienen como finalidad acondicionar los campos después de soportar el tráfico intenso de la maquinaria agrícola emplea- da en la cosecha. Se alcanza un número máximo de cuatro (4) pases. Con respecto a la aplicación de agroquímicos sobre el suelo, vale la pena mencionar que sólo se tendrá en cuenta la aplicación de fertilizantes sintéticosy orgánicos, dado que los herbicidas e insecticidas aplicados para el control de malezas, son responsables de toxicidad y contaminación, pero su contribución a las emisiones de GEI es casi nula y por tanto, para el indicador de Huella de Carbono es insignificante. (González \& Guerra, 2015)

En resumen, para efectos del proyecto de investigación propuesto, fue necesario delimitar las fuentes de emisión de dióxido de carbono y óxido nitroso proveniente de las labores de campo en análisis. De esta forma se determinó el comportamiento de la huella de carbono durante la preparación y levante de un cultivo de caña de azúcar. Principalmente, se evaluó la combustión fósil proveniente de la maquinaria agrícola empleada para preparar el suelo según el ciclo de cosecha (plantilla, soca), y realizar labores mecanizadas de levante del cultivo. Así como la descomposición de agro-químicos en suelos. A partir de esto, se desarrolló un diagrama de flujo con los límites del sistema a modelar (Figura 1).

\section{Aplicación y Discusión}

\subsection{Diagrama Causal de la situación global actual}

Con el objetivo de visualizar de forma sistémica el problema de investigacion abordado previamente, a continuación se presenta un diagrama de causalidad entre las variables; con la presencia de ciclos reforzadores que agravan la situación, así como otros ciclos compensadores que permiten aminorar los efectos colaterales producidos.

"La agricultura contribuye al cambio climático porque emite a la atmósfera cantidades significativas de dióxido de carbono $\left(\mathrm{CO}_{2}\right)$, metano $\left(\mathrm{CH}_{4}\right)$ y óxido nitroso $\left(\mathrm{N}_{2} \mathrm{O}\right)$. Actividades como el cultivo, la producción de los insumos utilizados durante el cultivo (por ejemplo, los fertilizantes químicos), el procesamiento, el almacenamiento, el envasado y la distribución de productos agricolas, emiten, todas ellas, GEI. El sector agrícola también está sufriendo directamente el efecto de los cambios que se están produciendo en nuestro clima, entre ellos, la frecuencia, cada vez mayor, de fenómenos meteorológicos extremos tales como 


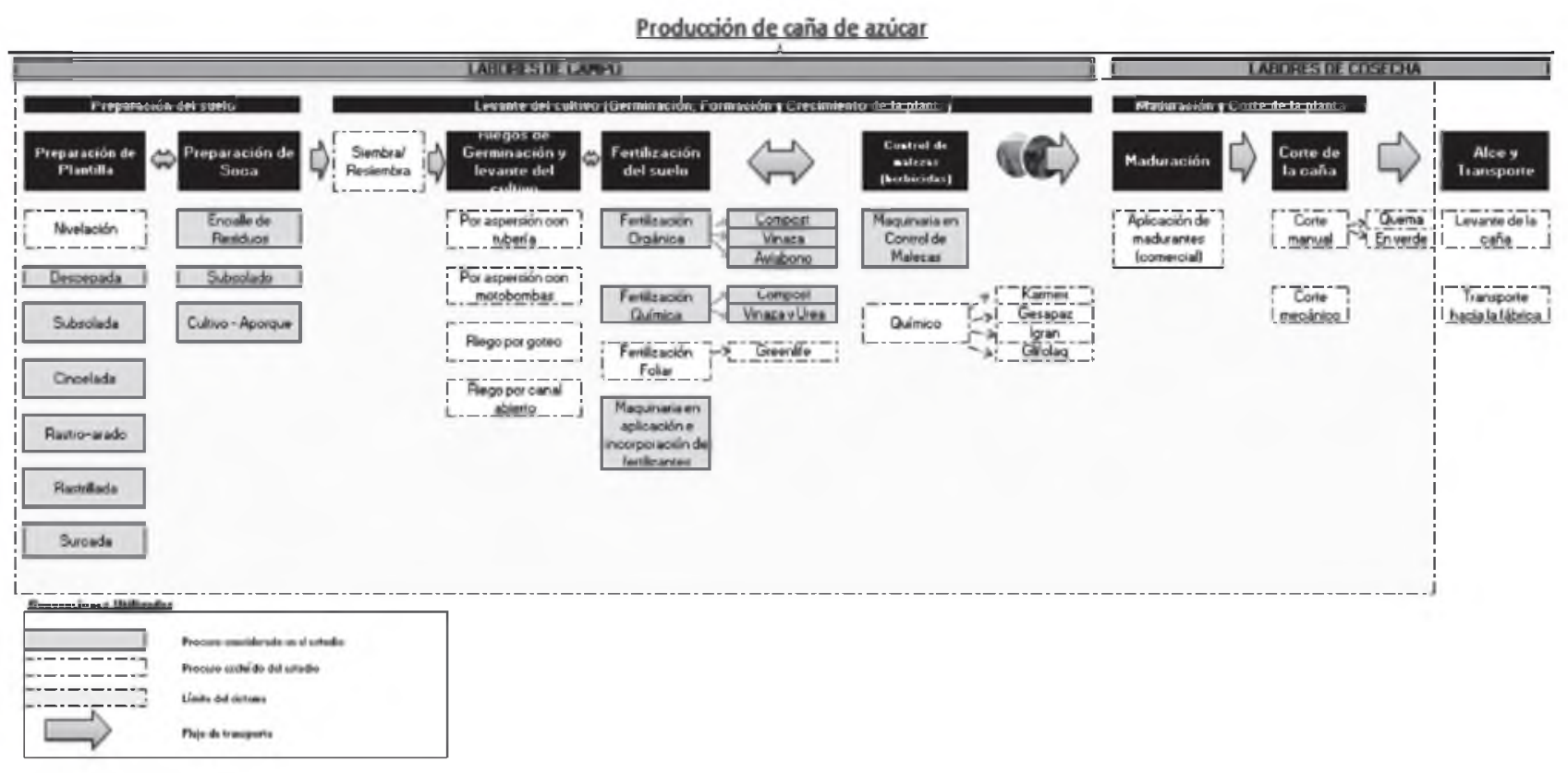

Figura 1. Diagrama de flujo con los límites del sistema modelado

tormentas, inundaciones y sequias, que acortan el periodo de crecimiento y merman los rendimientos " (ITC, 2012). Partiendo de esta relación recíproca de causa-efecto entre las actividades agrícolas y el cambio climático, en la Figura 2 se destacan dos ciclos reforzadores. En primer lugar, con la problemática del cambio climático a nivel mundial, consecuencia de la concentración de los gases de efecto invernadero en la atmósfera que elevan el nivel de temperatura ambiente y hace que se produzcan menores precipitaciones; ocasiona la erosión de los suelos, lo cual afecta negativamente el rendimiento de los cultivos y por tanto su productividad. En segundo lugar, a partir de una reducción de la productividad, los ingenios realizan ajustes en la programación de sus actividades para garantizar el desarrollo de la planta de caña de azúcar, con el objetivo de maximizar la producción por hectárea del cultivo. En la mayoría de los casos se toma la decisión de administrar al cultivo una dosis completa de fertilizantes en una sola aplicación y de realizar el tipo de preparación de suelo que mayor número de labores agrícolas exige. El uso intensivo de combustibles fósiles contribuye al impacto ambiental con la acumulación de $\mathrm{CO}_{2}$ en la atmósfera. Así mismo, el uso de fertilizantes, herbicidas, madurantes, entre otros, eleva el nivel de mineralización de los suelos, contribuyendo principalmente a la generación de $\mathrm{N}_{2} \mathrm{O}$.

Dada la creciente preocupación internacional por la mitigación del cambio climático que se ha disparado a causa de las emisiones de estos gases a la atmósfera; se destacan dos ciclos compensadores. En primer lugar, se han desarrollado iniciativas de medición, control y reducción de los mismos. Por ejemplo, con la firma del Protocolo de Kioto se desarrollaron un conjunto de políticas nacionales, como los mecanismos de desarrollo limpio (MDL), la política de adaptación y mitigación al cambio climático, y la política nacional de producción más limpia; entre otros. Además de las certificaciones sobre huella de carbono a nivel internacional (ISO 14064, ISO 14067), las cuales podrían representar grandes beneficios para las empresas colombianas; como el acceso libre al mercado internacional, así como poder diferenciar sus productos y segmentar sus mercados para un cliente cada vez más exigente y preocupado por conocer el indicador de huella de carbono de sus productos. Además cumplir con estas normatividades voluntarias actualmente, representa una preparación para cumplir con futuras medidas obligatorias. Obteniendo así la oportunidad de participar en mercados internacionales de carbono, uno de los tres mecanismos propuestos en el protocolo de Kyoto para reducir las emisiones de GEI. Por otro lado, con el objetivo de maximizar la productividad del cultivo con el menor impacto ambiental posible; es necesario considerar un nuevo sistema para administrar los recursos en campo y monitorear las actividades que se realizan sobre el cultivo, definiendo así el tratamiento específico y diferenciado que requiere el cultivo según las condiciones climáticas y características del mismo, el cual se conoce como "Agricultura de Precisión" 
(Grisso, Alley, McClellan, Brann, \& Donohue, 2009). La implementación de esta tecnología está diseñada para buscar la mejor opción que permita el ahorro en tiempo y costo de las actividades agrícolas, puesto que realiza control sobre las cantidades de fertilizante a aplicar sobre el cultivo, indica la entrega de nutrientes exactos en tasa variada y monitorea la calidad de aplicación y eficiencia de la maquinaria requerida para llevar a cabo estas labores. Esto permite compensar u aminorar los efectos tardíos del impacto ambiental, dado que brinda información técnica y consistente con mapas que determinan las condiciones ideales de operación para una adecuada toma de decisiones. Por ejemplo sobre la fracción de aplicación óptima de fertilizantes y labores agrícolas necesarias por el cultivo, lo cual permite operar sin desperdicio alguno (Bramley, 2009).

De esta forma, se puede observar la importancia de este estudio de cálculo y modelación de la huella de carbono con la planeación estratégica de los ingenios azucareros, que buscan determinar el impacto ambiental generado en campo, para establecer planes de mitigación que les permitan cumplir con los niveles de emisión exigidos, adquirir tecnologías más eficientes y bajas en carbono que al mismo tiempo les permitan optimizar el desarrollo del cultivo e incrementar los niveles de productividad; además de tener la oportunidad de expandir sus mercados a nivel internacional y participar activamente en éstos, ajustándose a sus normatividades.

\subsection{Diagrama Causal del problema de investi- gación local}

De la misma forma en que se desarrollo el diagrama causal de la situación problemática global, se definio desarrollar el diagrama causal presentado en la Figura 3 , el cual permite una visión sistémica local perteneciente al caso de estudio de análisis. Como bases para su construcción se plantearon las principales hipótesis dinámicas a validar en la investigación actual.

En primer lugar, se presentan las principales hipótesis que alimentan el diagrama causal:

- La fertilización química en los cultivos de caña de azúcar es la fuente principal de emisiones de óxido nitroso.

- La combustión fósil generada por la maquinaria agrícola en el cultivo de caña de azúcar es la fuente principal de emisiones de dióxido de carbono.

- Los residuos orgánicos que deja el cultivo después de la cosecha y los cuales son procesados en planta y aplicados de nuevo en el siguiente ciclo de cosecha como fertilizantes orgánicos, contribuyen a la generación de emisiones de $\mathrm{N}_{2} \mathrm{O}$ junto con los fertilizantes sintéticos nitrogenados, herbicidas y madurantes aplicados durante el levante del cultivo.

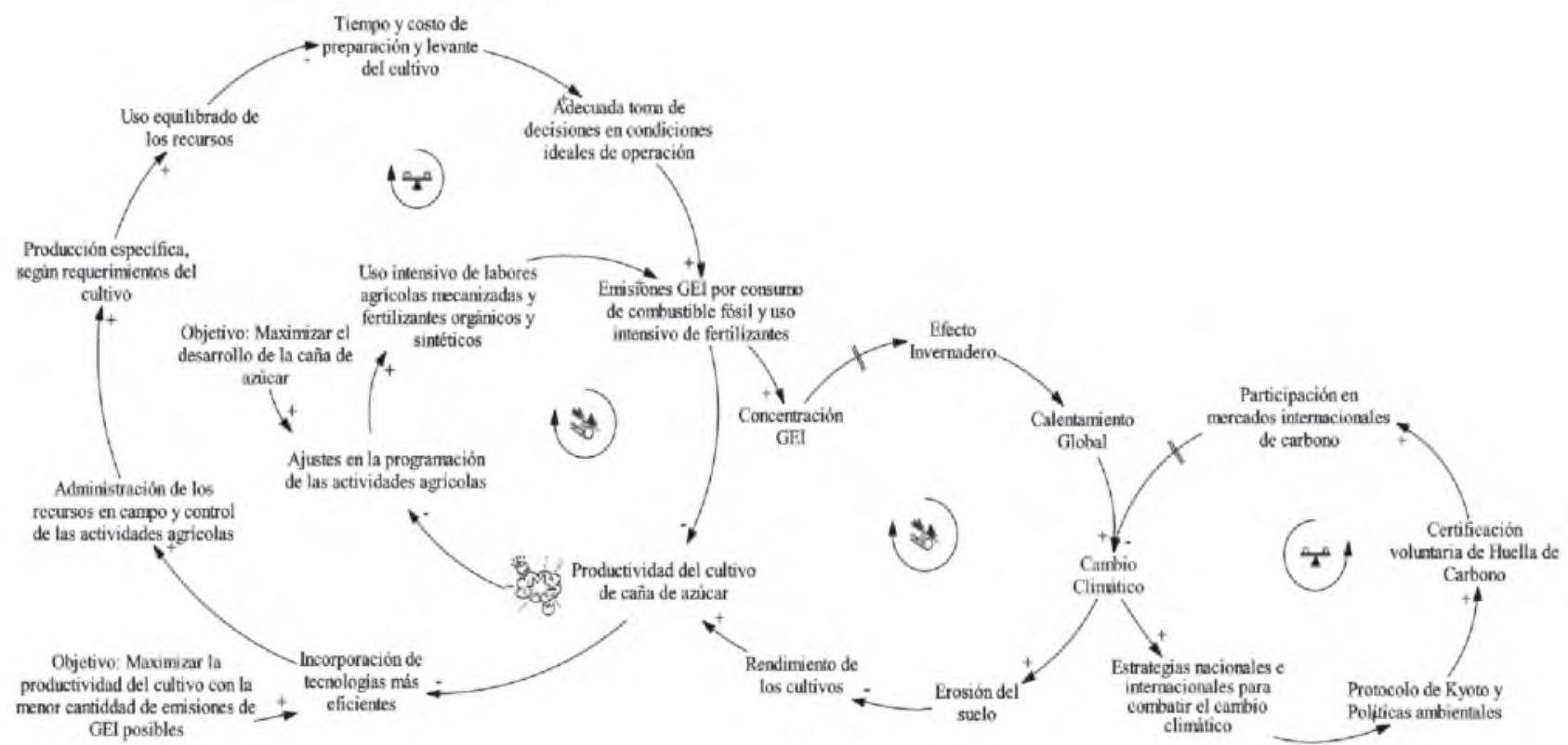

Figura 2. Diagrama Causal de la Huella de Carbono de un cultivo de caña de azúcar y estrategias de mitigación 
- La acumulación de GEI proveniente de la combustión fósil y los suelos gestionados hace que el sector agrícola sea uno de los principales responsables del Calentamiento Global, y por ende del efecto de Cambio Climático.

A continuación se presentan otras hipótesis dinámicas en las cuales no es posible observar una relación directa en el diagrama causal local (Figura 3), pero se deben considerar como escenarios a validar por medio de los análisis de sensibilidad realizados a los modelos.

- El cultivo en el ciclo de cosecha tipo Soca emite menos emisiones de GEI a la atmósfera, con respecto al cultivo en el ciclo de cosecha tipo Plantilla.

- El sistema de labranza liviana (Sistema de labranza que implica un menor número de labores mecánicas y un menor número de pases por labor realizada) genera menores emisiones de GEI con respecto al sistema de labranza convencional, durante la preparación del suelo tipo Plantilla.

- La utilización de fertilizantes sintéticos genera mayores niveles de emisiones de GEI con respecto a la utilización de fertilizantes orgánicos.

- La fertilización fraccionada y en diferentes edades de la caña permite una reducción del nivel de emisiones de GEI en campo.

El diagrama causal que describe la situación problema presenta dos ciclos reforzadores, a partir las dos fuentes de emisión a evaluar. Comenzando con la hipótesis de que se presenta una reducción en la cantidad de semillas sembradas, se obtendrá una menor área de caña de azúcar cultivada, y por tanto, una disminución en la cantidad de caña cosechada. Dado lo anterior, se reduce la cantidad de residuos orgánicos (bagazo, cachaza, vinaza) disponibles para producir los fertilizantes orgánicos. Esto conduce a una disminución en la productividad del cultivo. Ante esto, aumenta la aplicación intensiva de fertilizantes, herbicidas y madurantes nitrogenados sobre el cultivo, ocasionando emisiones de óxido nitroso a la atmósfera. Por otro lado, se incrementa el uso de maquinaria agrícola, y con éste el consumo de energía, específicamente combustible (Diesel, Gasolina) proveniente de las fuentes móviles, y electricidad procedente de las fuentes fijas. Estas labores mecanizadas producen emisiones de dióxido de carbono a la atmósfera. De esta forma, la acumulación de estas moléculas de gases contribuye al efecto invernadero, y por ende al calentamiento global del planeta, el cual a su vez influye en el cambio climático. Por tanto, la presencia de fenómenos meteorológicos extremos impacta negativamente la producción de caña de azúcar, reforzando el sistema de nuevo que busca intensificar las labores agrícolas para incrementar y mantener los niveles de producción, a expensas de la degradación ambiental en el largo plazo.

\section{Fases del proyecto de investigación futuro}

En concurso del presente proyecto, se ha resuelto la primera fase de investigación, así como la primera parte de la segunda fase, relacionada con la descripción de la estructura metodológica requerida para una definición formal de los modelos de simulación que abarcaran las fuentes de emisiones de GEI en cada etapa del cultivo. El proceso futuro a realizar comprende la formulación de dos modelos de emisiones de GEI a partir de las dos fuentes principales de emisión identificadas en la preparación de los suelos y levante del cultivo, como son la combustión fósil por parte de la maquinaria agrícola, y la descomposición de los fertilizantes aplicados sobre el suelo. Igualmente, comprende las siguientes dos etapas previamente referenciadas, las cuales son validación de los modelos y análisis de resultados.

Conocidos los alcances de este estudio y la importancia de desarrollar modelos dinámicos de simulación del comportamiento de las emisiones de GEI generadas en los cultivos de caña de azúcar, que permitan administrar de forma eficiente los recursos y desarrollar prácticas agrícolas más limpias y sostenibles en el tiempo, se recomienda validar las siguientes hipótesis dinámicas en proyectos externos de investigación.

- En la etapa de preparación de suelos y levante del cultivo el nivel de emisiones de GEI es mayor con respecto a las siguientes etapas de mantenimiento del mismo, debido a la concentración de labores requeridas en los primeros meses de ciclo de vida del cultivo.

- Las variables meteorológicas y climatológicas como temperatura, precipitación y presión atmosférica afectan el nivel de emisiones de GEI acumulado en la atmósfera. 


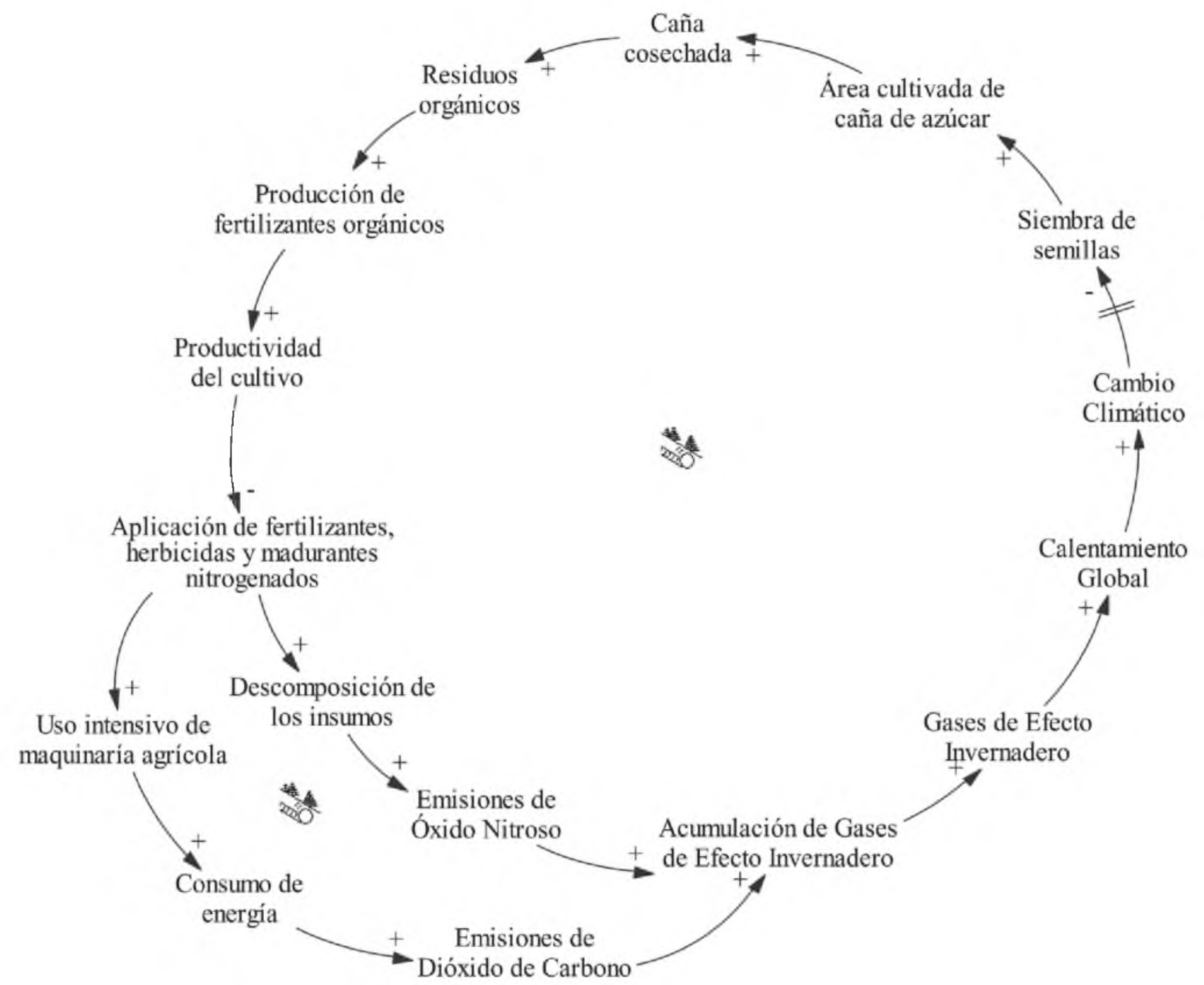

Figura 3. Diagrama Causal del problema de investigación.

- Las cantidades aplicadas de fertilizantes y el uso intensivo de las labores agrícolas dependen de las condiciones climáticas, características del cultivo y la productividad del mismo.

- La planta de caña de azúcar absorbe $\mathrm{CO}_{2}$ presente en la atmósfera y éste varía según el ciclo de vida del cultivo (Germinación, Formación, Crecimiento, Maduración).

Fuera del alcance de este estudio, como proyecto externo de investigación se recomienda desarrollar modelos de simulación que permitan establecer metas de reducción del nivel de emisiones de GEI en la atmosfera, en común acuerdo con asociaciones internacionales sobre cambio climático. Con el objetivo de realizar análisis de sensibilidad que permitan modificar las metas establecidas, y establecer niveles máximos de emisión para el sector agrícola, sin afectar la productividad del cultivo. Esto le permitirá a los ingenios establecer planes de reducción de las emisiones en el largo plazo, y evaluar periódicamente el impacto de sus actividades agrícolas sobre el cultivo.

\section{Conclusiones}

La definición de una estructura metodológica desde el punto de vista de la dinámica de sistemas, permite abordar problemas de investigación de diversa índole de manera sistémica.

Específicamente, por medio de los diagramas causales empleados como herramienta fundamen- 
tal en el establecimiento de los modelos dinámicos de simulación propuestos, es posible identificar diferentes bucles de retroalimentación en el sistema. Los bucles positivos conllevan a un ciclo reforzador. Esto ocasiona una situación inestable, por su comportamiento dinámico de crecimiento, evolución y posible colapso del sistema. En la aplicación local presentada, esta situación se observó en la problemática de cambio climático y sus efectos colaterales negativos sobre la productividad del cultivo. Por otro lado, se pueden presentar bucles de retroalimentación negativos, los cuales conducen a un ciclo compensador. De esta forma, se generan fuerzas de resistencia que limitan el crecimiento y tienden a estabilizar el sistema. En este caso, a nivel internacional se han desarrollado iniciativas mundiales de medición, control y reducción de los gases de efecto invernadero. Específicamente estos son: los mecanismos de desarrollo limpio, la política de adaptación y mitigación al cambio climático y la política nacional de producción más limpia, los cuales tienen como objetivo aminorar los efectos colaterales producidos por el cambio climático.

La identificación de estructuras causales de retroalimentación de la situación problema analizada, facilita la comprensión del comportamiento del sistema real y por tanto, permiten una adecuada formulación de los modelos dinámicos propuestos. Lo anterior facilita el proceso de toma de decisiones sobre prácticas agrícolas de operación, en un entorno ambiental complejo en constante crecimiento.

\section{Referencias bibliográficas}

Bramley, R. G. V. (2009). Lessons from nearly 20 years of Precision Agriculture research, development, and adoption as a guide to its appropriate application. Crop and Pasture Science, 60(3), 197. http://doi.org/ $10.1071 / \mathrm{CP} 08304$

Carbonell, J. A., Quintero, R., Stember, J., Osorio, C. A., Isaacs, C., \& Victoria, J. I. (2011). Zonificación Agroecológica para el Cultivo de Caña de Azúcar en el Valle del río Cauca (tercera aproximación). Cali.

Concha, A. M., \& Rojas, J. L. (2011). Diseño de Experimento para el cálculo teórico de emisiones de $\mathrm{CO}_{2}$ generadas por cuatro tipos de preparación del suelo para un cultivo de caña de azúcar. Universidad Icesi.
Conway, G. R., \& Barbie, E. B. (1988). After the Green Revolution. Futures, 20(6), 651-670. http://doi.org/ 10.1016/0016-3287(88)90006-7

De Klein, C., Novoa, R., Ogle, S., Smith, K., Rochette, P., \& Wirth, T. (2006). Capítulo 11: Emisiones de $\mathrm{N}_{2} \mathrm{O}$ de los suelos gestionados $\mathrm{y}$ emisiones de $\mathrm{CO}_{2}$ derivadas de la aplicación de Cal y Urea. In Directrices del IPCC de 2006 para los inventarios nacionales de gases de efecto invernadero (pp. 11.1-11.56 Vol. 4). Hayama, Japón: IGES.

Forrester, J. W. (1961). Industrial dynamics. (MIT, Ed.). Cambridge, MA.

Gómez, D., \& Watterson, J. (2006). Capítulo 2: Combustión Estacionaria. In Directrices del IPCC de 2006 para los inventarios nacionales de gases de efecto invernadero (Vol. 2, pp. 2.1-2.47). Hayama, Japón.

González, O., \& Guerra, A. (2015). La Huella de Carbono del Azúcar de Guatemala, Zafra 2013-2014. Revista Atagua.

Grisso, R., Alley, M., McClellan, P., Brann, D., \& Donohue, S. (2009). Precision Farming. A Comprehensive Approach. Virginia Cooperative Extension, 442-500.

ITC. (2012). Normas de la huella de carbono de productos agrícolas. Ginebra, Suiza.

Liévano, F., \& Londoño, J. E. (2012). El pensamiento sistémico como herramienta metodológica para la resolución de problemas. Revista Soluciones de Postgrado EIA, 4(8), 43-65.

López, A., Rodríguez, L. M., Lubo, C. M., Arenas, F. A., \& Sierra, B. E. (2014). Evaluating Carbon Footprint Behavior in the Agriculture and Energy Sectors?: A Review. Sistemas \& Telemática, 12, 35-53.

Mclaughlin, M., Tiller, K., Naidu, R., \& Stevens, D (1996). Review: the behaviour and environmental impact of contaminants in fertilizers. Australian Journal of Soil Research, 34(1), 1. http://doi.org/ 10.1071/SR9960001

Rapal Uruguay. (2010). Impactos del modelo de agricultura industrial. Contaminación y eutrofización del agua. Montevideo, Uruguay. 
Sterman, J. D. (2000). Business dynamics: systems thinking and modeling for a complex world (Irwin/ $\mathrm{McGr})$. Boston, MA.

Waldron, C., Harnisch, J., Lucon, O., Mckibbon, R., Saile, S., Wagner, F., \& Walsh, M. (2006). Capítulo 3: Com- bustión móvil. In Directrices del IPCC de 2006 para los inventarios nacionales de gases de efecto invernadero (pp. 3.1-3.78 Vol. 2). Hayama, Japón: IGES.

Zoratto, A. C. (2006). Main Impacts of Sugar Cane. Virtual Pro. 
